\title{
Low-energy excitations of double quantum dots in the lowest Landau level regime
}

\author{
N. Barberán and J. Soto \\ Departament d'Estructura i Constituents de la Matèria Facultat de Física, Universitat de Barcelona, E-08028 Barcelona, \\ Catalonia, Spain
}

(Received 18 April 2002; published 27 November 2002)

\begin{abstract}
We study the spectrum and magnetic properties of double quantum dots in the lowest Landau level for different values of the hopping and Zeeman parameters by means of exact diagonalization techniques in systems of $N=6$ and 7 electrons and a filling factor close to 2 . We compare our results with those obtained in double quantum layers and single quantum dots. The Kohn theorem is also discussed.
\end{abstract}

DOI: 10.1103/PhysRevB.66.205325 PACS number(s): 73.21.-b, 73.43.-f, 73.21.La, 73.43.Lp

\section{INTRODUCTION}

The understanding of the structure and properties of double quantum dots (DQD's) grown on the perpendicular direction to their plane, and subject to an external constant magnetic field $\vec{B}$, has attracted special interest due to the wealth of quantum states that are actually realized. This wealth does not only refer to the ground states (GS's), which provide a quite intricate phase diagram, ${ }^{1,2}$ but also to the variety of different excited states. This allows one to have a large quantity of different sets of properties of the DQD system that can model, for instance, point contacts within a device built for electron transport.

Special interest is given to the regime for which the magnetic field is so strong that only the lowest Landau level (LLL) is occupied but not strong enough to prevent any spin polarization. We will always assume that the second Landau level is far enough away that we can ignore any mixture between Landau levels. In the symmetric gauge, the projection of the DQD Hamiltonian to the LLL is given by ${ }^{3}$

$$
H=\alpha M+\beta N-\Delta_{z} S_{z}-\Delta_{t} X+H_{\text {int }},
$$

where

$$
\alpha=\frac{\hbar}{2}\left[\sqrt{\omega_{c}^{2}+4 \omega_{0}^{2}}-\omega_{c}\right]
$$

and

$$
\beta=\frac{\hbar}{2} \sqrt{\omega_{c}^{2}+4 \omega_{0}^{2}}
$$

$\omega_{c}=e B / \mathrm{cm}^{*}$ is the cyclotron frequency, $m^{*}$ being the effective electron mass in the semiconductor host, and $e$ and $c$ are the electron charge and the speed of light in vacuum, respectively. The frequency associated with the parabolic confining potential in both dots is given by $\omega_{0}, M$ is the total angular momentum and $N$ is the total number of electrons. The Zeeman coupling is given by $\Delta_{z}=g \mu_{B} B$, with $g$ the Landé $g$ factor and $\mu_{B}$ the Bohr magneton (for the free electron mass, $\left.\mu_{B}=e \hbar / 2 m c\right)$. The single particle energy gap between symmetric $(s)$ and antisymmetric (a) states, combinations of $|r\rangle$ and $|l\rangle$ single particle states of electrons confined in the right and left dot, respectively (see below), is given by $2 \Delta_{t}$, and $X=N_{s}-N_{a}$ is the balance between symmetric and antisym- metric single particle states. Finally, $H_{i n t}$ is the Coulomb interaction term. Hereafter all distances will be given in units of the magnetic length, defined as

$$
l_{B}=\left[\frac{\hbar}{m^{*}\left(\omega_{c}^{2}+4 \omega_{0}^{2}\right)^{1 / 2}}\right]^{1 / 2},
$$

and all energies in units of $e^{2} /\left(\epsilon l_{B}\right), \epsilon$ being the dielectric constant of the semiconductor host. The Zeeman energy, the tunneling term scaled by $\Delta_{t}$, the kinetic contribution given by $\alpha$ and $\beta$ and the Coulomb interaction provide the ingredients of the system. The applied magnetic field can be directed in any direction in space in such a way that its action has different effects on the kinetic contribution in which only the component along the $Z$ direction plays a role and in the Zeeman effect to which the total magnetic field contributes. The eigenstates of the Hamiltonian are characterized by the total angular momentum $M$, the total spin along the $\vec{B}$ direction given by $S_{z}$, and the parity $P .{ }^{1,4}$ These parameters are related to the invariance of the Hamiltonian under space rotations along the $Z$ direction, rotations of spin and specular reflection in the plane interdots, respectively. ${ }^{1}$ We will denote by $\left(M, S_{z}, P\right)$ the configuration that determines each separated subspace of eigenstates.

Previous studies in DQD's based on exact diagonalizations, on the one hand, ${ }^{1,2}$ and the large experience extracted from mean field (MF) approximations and effective field theories in double layer (DL) systems, on the other hand, ${ }^{5-9}$ have provided a quite complete picture of the GS phase diagram. It is well established in both DL (Ref. 7) and DQD (Ref. 1) systems that the variation of $\Delta_{t}$, leaving all the other parameters fixed, induces several changes in the GS. For $\Delta_{z} \gg \Delta_{t}$ the GS is ferromagnetic $|\mathrm{FM}\rangle$ with symmetric and antisymmetric single particle states equally populated and with all the electrons in spin up states. In contrast for $\Delta_{z}$ $\ll \Delta_{t}$ the GS is symmetric $|\mathrm{SYM}\rangle$ with all the electrons occupying symmetric single particle states with up and down spins equally populated. In between, for comparable values of Zeeman and tunneling energies, intermediate states of the type called canted states $(|C\rangle)$ become GS's. The canted state is the GS solution between the $|F M\rangle$ and $|S Y M\rangle$ in a MF calculation for a DL system and it is characterized by a ferromagnetic order in the direction perpendicular to the layers and antiferromagnetic order in plane ${ }^{7}$. 
A remarkable result of the Hartree-Fock (HF) approximation for DL quantum Hall systems at a filling factor $\nu=2$ is that there are only three possible different GS's: $|\mathrm{FM}\rangle,|C\rangle$, and $|\mathrm{SYM}\rangle$ distributed over a universal phase diagram which boundaries depend only on three energy scales whereas, the Hamiltonian depends on four independent energy scales. ${ }^{7}$

Certain ground states obtained from exact diagonalization in DQD's have also been identified as canted states since their overlap with the MF canted GS's for finite systems, projected on the appropriate subspace with well defined $S_{z}$, is nearly $1 .{ }^{1}$ The total number of different canted states between $|\mathrm{FM}\rangle$ and $|\mathrm{SYM}\rangle$ in a DQD depends on the number of electrons. As a general rule, keeping fixed $\Delta_{z}$ and increasing $\Delta_{t}$, the GS evolves as

$$
\begin{aligned}
|\mathrm{FM}\rangle & \rightarrow\left(M, S_{z}, P\right) \rightarrow\left(M, S_{z}-1,-P\right) \\
& \rightarrow\left(M, S_{z}-2, P\right), \ldots,|\mathrm{SYM}\rangle .
\end{aligned}
$$

A simultaneous change of spin and parity occurs for the GS in each phase transition, whereas the total angular momentum remains unchanged. ${ }^{1}$

No direct information about the in-plane order has been obtained so far from the exact solutions due to the fact that they have quantum numbers $\left(M, S_{z}, P\right)$ that are well defined. No information can be directly obtained from order parameters that depend on single operators of the type $S_{x}^{R}$ or $S_{x}^{L}$, since their expectation values vanish. However, indirect information about the order in plane can be obtained from the properties of the excited states as we will show in the discussion of Figs. 1 and 2 below. Within the great number of possible low energy excitations that provide information about the properties of the system, we will draw our attention to those related to three main different points.

First, we search for particlelike excitations independent of the electron-electron interaction and so independent of the number of electrons. We discuss when the Kohn theorem ${ }^{10}$ holds in a parabolic DQD system (as is always the case for a single parabolic QD).

Second, we look for the types of excitations that soften as they come close to a GS transition that takes place with the variation of some input parameter. These excitations provide a clear and easy way to map the GS phase diagram.

Third, in the last point we concentrate on the dispersion relation $\omega(l)$ of two different type of excitations, one with and the other without a simultaneous spin and isospin flip (see below). In a single QD the Coulomb contribution to the dispersion relation of excitations over a ferromagnetic GS decreases with angular momentum due to expansion. This is the general behavior, except at "magic" values of $l^{*},{ }^{11}$ for which the system increases in angular momentum from $l^{*}$ to $l^{*}+1$ but does not increase in size; hence the Coulomb contribution remains constant. These magic values of $l$ are related to the incompressible states. Our aim is to see if there is a similar behavior in the DQD system.

This paper is organized as follows: In Sec. II we make a brief account of the exact diagonalization used in our calculations. In Sec. III we present the outstanding features of some excited states of different multipolarity over different GS's, paying special attention to the different behavior of even and odd systems. We will follow the three main points previously mentioned. Finally in Sec. IV we draw our conclusions.

\section{EXACT DIAGONALIZATION}

Exact diagonalization can be performed in separate boxes for different configurations characterized by $\left(M, S_{z}, P\right)$. Each configuration determines a finite-eigenstate subspace. Within the LLL regime, in all expansions we will use noninteracting single particle wave functions, which do not have nodes along the radius and are given by

$$
\Phi_{m}(\vec{r}, \sigma, \Lambda)=\phi_{m}(\vec{r})|\sigma \Lambda\rangle,
$$

where $m$ is the single particle angular momentum, $\Lambda=r, l$, $\sigma=\uparrow, \downarrow, \vec{r}=(x, y)$, and $\phi_{m}(\vec{r})$ are the Fock Darwin wave functions given by ${ }^{11}$

$$
\phi_{m}(\vec{r})=\frac{1}{\sqrt{2 \pi m ! 2^{m}}} e^{-i m \theta} r^{m} e^{-r^{2} / 4}
$$

Along the $Z$ direction we assume delta charge distributions separated by $d$, the distance between the dots (we consider $d=1$ in all numerical performances).

Within our calculations we will use symmetric $|s\rangle$ and antisymmetric $|a\rangle$ single particle states, related by $|r\rangle$ and $|l\rangle$ by

$$
|s\rangle=\frac{1}{\sqrt{2}}(|r\rangle+|l\rangle)
$$

and

$$
|a\rangle=\frac{1}{\sqrt{2}}(|r\rangle-|l\rangle)
$$

we will use the term isospin referred to this degree of freedom (sometimes referred as pseudospin in literature; see Ref. 5). None of the parameters $r$ and $l$ or $s$ and $a$ are well defined quantum numbers since the Coulomb interaction mixes $s$ and $a$ and the tunneling process mixes $r$ and $l$. The well established restriction is that parity must be preserved; this means that the change in symmetry $(s \rightarrow a$ or $a \rightarrow s$ ) due to electron interaction must take place by pairs of electrons, and never by one alone (see below).

We will consider excited states over the three possible types of GS's, and choose the parameters in such a way that for an even number of electrons in the ferromagnetic GS we have a filling factor $\nu=2$. We proceed as follows. Once the input parameters are fixed, we determine first the finite number of Slater determinants built up from single particle wave functions of the type given by Eq. (6) which provide bases for each subspace configuration $\left(M, S_{z}, P\right)$. Then the diagonalization of the Hamiltonian given by Eq. (1) is straightforward, except for the Coulomb term. Although the Coulomb interaction does not mix $|r\rangle$ and $|l\rangle$ single particle states, some manipulations must be done in order to operate over $|s\rangle$ and $|a\rangle$ wave functions. In a second quantization, the interaction Hamiltonian is given by 


$$
H_{\text {int }}=\frac{1}{2} \sum_{i j k l}\left\langle\phi_{i} \phi_{j}|V| \phi_{k} \phi_{l}\right\rangle a_{i}^{+} a_{j}^{+} a_{l} a_{k},
$$

where $V$ is the Coulomb interaction and the subindexes denote angular momentum $m=0,1,2, \ldots$, spin $=\uparrow, \downarrow$, and isospin $\tau=a, s$. Taking into account that the single particle wave functions are related by Eqs. (8) and (9) and that the right and left single states are $\delta$ distributions of types $\delta(z)$ and $\delta(z-d)$, respectively, it is easy to show that

$$
\phi_{a}^{*} \phi_{a}=\phi_{s}^{*} \phi_{s} \sim \frac{1}{2}[\delta(z)+\delta(z-d)]
$$

and

$$
\phi_{s}^{*} \phi_{a} \sim \frac{1}{2}[\delta(z)-\delta(z-d)]
$$

for each electron. As a consequence, there are only three possibilities for the expectation values of $V$ : (i) the interacting electrons do not change their isospin as in

$$
\left\langle\phi_{s} \phi_{s}|V| \phi_{s} \phi_{s}\right\rangle=\frac{1}{2}\left(\left\langle V_{r r}\right\rangle+\left\langle V_{r l}\right\rangle\right)
$$

(ii) only one electron changes its isospin as in

$$
\left\langle\phi_{s} \phi_{a}|V| \phi_{a} \phi_{a}\right\rangle=0
$$

and (iii) both electrons change their isospin index as in

$$
\left\langle\phi_{s} \phi_{a}|V| \phi_{a} \phi_{s}\right\rangle=\frac{1}{2}\left(\left\langle V_{r r}\right\rangle-\left\langle V_{r l}\right\rangle\right)
$$

In the brackets on the right hand side of these equations, the integral over the $Z$ coordinate has been performed, and the potentials are given by

$$
V_{r r}=V_{l l}=\frac{e^{2}}{\epsilon r}, \quad V_{r l}=V_{l r}=\frac{e^{2}}{\epsilon\left(r^{2}+d^{2}\right)^{1 / 2}}
$$

with $r=\left|\vec{r}_{1}-\vec{r}_{2}\right|, 1$ and 2 being the two interacting electrons. That is to say the Coulomb interaction either leaves the isospin of the electrons unchanged, if it operates with

$$
V_{0}=\frac{1}{2}\left(V_{r r}+V_{r l}\right)
$$

or changes the isospin of two electrons if it operates with

$$
V_{1}=\frac{1}{2}\left(V_{r r}-V_{r l}\right),
$$

in such a way that parity $P$, given by $P=(-1)^{X / 2}$ is preserved $\left(X=N_{s}-N_{a}\right) .{ }^{1}$ The change of isospin of a single electron is forbidden.

\section{MULTIPOLAR EXCITATIONS}

Guided by an interest in the properties of the multipole mode excitations and also by an interest in those excitations that soften at the boundaries of the phase transitions within the phase diagram, we explored the eigenenergies and eigenstates coming out of exact diagonalizations. We consider, in general, the multipolar excitations characterized by $\omega(l)$ with or without a simultaneous change of spin and/or parity, and in some cases we concentrate on $l=0$ or 1 .

Before focusing on the first point, that is, within the search of excitations which keep the internal Coulomb energy constant, let us briefly comment on the Kohn theorem for a DQD. The Kohn theorem for a single QD states ${ }^{12}$ that in the process of absorption (or emission) of a photon of wavelength much larger than the radius of the dot confined by a parabolic potential, the initial and final electronic states can differ from one another only by the center of mass (CM) excitation. The number of confined electrons and the interaction between them has no influence on the values of resonance energies. For a DQD, however, qualifications to this statement may be required for different directions of the incident electric field with respect to the plane of the dots. It is easy to see that for homogeneous in-plane electric fields the Kohn theorem also holds for DQD's. However, this is not the case for homogeneous electric fields along the $Z$ direction, as we argue below.

Taking into account Eqs. (8) and (9) and the expression of the exciting potential (associated with $E_{\perp}$ directed along the $Z$ direction),

$$
H_{E_{\perp}} \sim \sum_{i=1}^{N} z_{i} E_{\perp}
$$

it is easy to see that $\left\langle\phi_{s}\left|H_{E_{\perp}}\right| \phi_{a}\right\rangle \sim d \neq 0$. That is to say, the operator $H_{E_{\perp}}$ changes the parity of the system and so $\left[H_{E_{\perp}}, H_{\text {int }}\right] \neq 0$ since the eigenstates of $H_{\text {int }}$ have a well defined parity. The part of the Hamiltonian which depends on the relative coordinates only receives other contributions aside from the interaction Hamiltonian (coming from the kinetic and from the tunneling terms ${ }^{11}$ within the first quantized expression); however, the possibility that the last bracket can be compensated for by the brackets of the other terms can be disregarded as they depend on different independent parameters. An alternative reasoning is presented in the Appendix. In the analysis of the exact diagonalization results which follows, we will verify, among other things, the applicability of the Kohn theorem.

Let us begin with the $N=7$ electron system, and consider first the excitations of the $|\mathrm{FM}\rangle$ GS that increase the angular momentum in one unit and leave all the other parameters of the configuration unchanged; that is to say, we consider,

$$
\left(M, S_{z}, P\right) \rightarrow\left(M+1, S_{z}, P\right) .
$$

The system jumps from a one-dimensional (1D) space configuration to a $2 \mathrm{D}$ space. The result is the excitation of the CM by $\alpha$ leaving the internal Coulomb energy unchanged. This is the well known intra-Landau-level dipole excitation whose energy decreases when the magnetic field increases that is, the $\omega_{-}=\alpha$ far-infrared resonance. ${ }^{11}$ No inter-Landaulevel transition of energy given by

$$
\omega_{+}=\frac{1}{2}\left[\sqrt{\omega_{c}^{2}+4 \omega_{0}^{2}}+\omega_{c}\right]
$$


is possible within the LLL considered in our calculation. Other Coulomb-independent interaction excitations are possible in the $N=7$ system. If we also allow changes in parity, that is to say if we consider

$$
\left(M, S_{z}, P\right) \rightarrow\left(M+1, S_{z},-P\right),
$$

the CM is excited with an energy $\alpha+\Delta_{t}$ (it is a CM excitation since the same result would be obtained for $N=1$ ). The previous case (of energy $\alpha$ ) would correspond to the excitation made by a nearly constant electric field (we are assuming dipole approximation) directed along the $X$ direction $\left(E_{\|}\right)$ and the last one (of energy $\alpha+\Delta_{t}$ ) would correspond to an electric field with an additional nonvanishing component along the $Z$ direction (let us call it $\mathbf{E}_{x z}$ ). Incidentally, this result can be used to determine experimentally the relative orientation of the DQD respect to the incident beam. The system absorbs a photon of energy $\alpha+\Delta_{t}$ with a maximum probability when the angle between the direction of the incident electric field and the normal to the plane of the DQD is $\theta=\pi / 4$.

For both excitations, with and without parity change, the system goes from a 1D space $(|\mathrm{FM}\rangle)$ to a 2D space of excited states. The CM excitation leaves the system in the higher energy state within the excited 2D configuration in such a way that

$$
E_{2}\left(M+1, S_{z}, \pm P\right)=E_{1}\left(M, S_{z}, P\right)+\alpha+(1 \mp 1) \Delta_{t} / 2
$$

and

$$
E_{1}\left(M+1, S_{z}, \pm P\right)<E_{2}\left(M+1, S_{z}, \pm P\right)
$$

where the eigenenergies $E_{i}$ have been ordered from lower to higher energy within each subspace. See Table I (cases $A$ and $B)$. The difference in energies in the excited configurations come from the decrease of Coulomb interaction due to the expansion of the eigenstate of lower energy. The highest energy eigenstate is a compact state and does not change in size. The two excitations of case $A$ with and without Coulomb contribution are equivalent to the "sum mode" and the "difference mode" found by Girvin and MacDonald ${ }^{6}$ for DL (without tunneling or Zeeman terms and for filling factor $\nu$ $=1 / 2$ ) within a single-mode approximation. Once one knows the decrease of energy of Coulomb origin from $E_{2}(10,7 / 2,0)-E_{1}(10,7 / 2,0)=E_{c}=0.0998$, one can distinguish in the $(11,7 / 2,0)$ configuration the double dipole $E_{6}$ $=E_{1}(9,7 / 2,0)+2 \alpha=16.0802(\alpha=0.2)$ from a quadrupole excitation given by $E_{4}=E_{1}(9,7 / 2,0)+2 \alpha-E_{c}=15.9803$. Surprisingly, the appearance of this eigenenergy in the output means that an eigenstate which is not a compact state nor a fully expanded state is a possible realization. The full expansion would imply a larger value of $E_{c}$ as $\Delta M=2$.

However, not all the previous scenario is reproduced in the $N=6$ system. If by analogy we consider the excitations $\left(M, S_{z}, P\right) \rightarrow\left(M+1, S_{z}, \pm P\right)$ from the $|\mathrm{FM}\rangle \mathrm{GS}$, the energy $E_{2}\left(M+1, S_{z}, P\right)=E_{1}\left(M, S_{z}, P\right)+\alpha$ is obtained according to the behavior of the $N=7$ system but no trace of the $\Delta_{t}$ energy $\left(\Delta_{t}=0.06\right)$ is present in $E_{2}\left(M+1, S_{z},-P\right)$.
TABLE I. Eigenenergies obtained from different configurations. We considered $\Delta_{t}=0.07$ and 0.06 for $N=7$ and 6, respectively; in both cases $\alpha=0.2, \beta=1.4$, and $\Delta_{z}=0.02$ and the GS is ferromagnetic. $A$ is the excitation due to an electric field given by $\vec{E}=E_{\|} \hat{i}$, and $B$ due to $\vec{E}=\vec{E}_{x z}$ being $\hat{i}$ the unitary vector along the $X$ axis. $C$ is due to an electric field expanded up to the quadrupolar approximation and directed along the $X$ axis.

\begin{tabular}{lccc}
\hline \hline \multicolumn{3}{c}{$N=6$} & \\
GS & $A$ & & \\
& $(7,3,0)$ & $(7,3,1)$ & $(8,3,0)$ \\
$(6,3,0)$ & $E_{1}=12.7868$ & $E_{1}=12.7232$ & $E_{1}=12.8804$ \\
$E_{1}=12.6802$ & $E_{2}=12.8802$ & $E_{2}=12.8434$ & $E_{2}=12.8915$ \\
& & & $E_{3}=12.9438$ \\
& & & $E_{4}=12.9868$ \\
& & & $E_{5}=13.0802$ \\
& & & \\
GS & & & \\
\hline$(9,7 / 2,0)$ & $(10,7 / 2,0)$ & $(10,7 / 2,1)$ & $(11,7 / 2,0)$ \\
$E_{1}=15.6802$ & $E_{1}=15.7803$ & $E_{1}=15.8503$ & $E_{1}=15.8700$ \\
& $E_{2}=15.8802$ & $E_{2}=15.9502$ & $E_{2}=15.8906$ \\
& & & $E_{3}=15.9523$ \\
& & & $E_{4}=15.9803$ \\
& & & $E_{5}=16.0231$ \\
& & & $E_{6}=16.0802$ \\
\hline \hline
\end{tabular}

From the analysis of the results of the $N=7$ system, one would suspect that the CM excitations (of energies $\alpha$ or $\alpha$ $\left.+\Delta_{t}\right)$ are a consequence of the Kohn theorem in the DQD system, in agreement with our assumption of a parabolic confining potential and the dipole-excitation approximation. However, if this were the case, the $N=6$ system would behave in the same way. The reason for the difference is provided by the different action of the electric fields that excite the system. The in-plane field produces a global shift of the system which does not affect the internal electron-electron interaction, and the CM absorbs the total energy $\alpha$. In contrast, if the external field has a nonvanishing component along the $Z$ direction, aside from the global absorptions of energy $\alpha$, an extra amount of energy is involved in the tunneling process. In the odd electron system, the unpaired electron tunnels from one dot to the other. This change, however, does not modify the electronic distribution asymmetry which was already present in the GS, and consequently does not modify the Coulomb interaction. On the other hand, in the even electron system due to the electronic jump, a change is produced between a symmetric distribution to a nonsymmetric one, necessarily giving a Coulomb contribution to the final energy. That is to say, the excitations induced by $E_{\perp}$ on an odd number of electrons do not modify the Coulomb energy. However, this is not a consequence of the Kohn theorem, as one may erroneously conclude.

Within the second main point, the search of excitations that mark the phase transitions, we concentrate on the evolution of the system as $\Delta_{t}$ increases, leaving all the other parameters unchanged. The appropriate excitations turned 


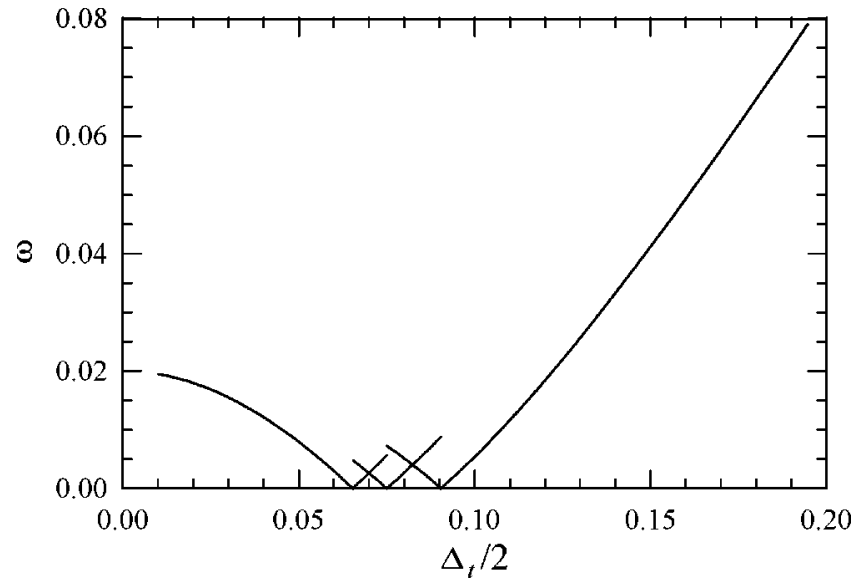

FIG. 1. Lowest energy excitation for $N=6$ as a function of $\Delta_{t}$ for different regions of the phase diagram. The excited configurations are (from left to right) $(6,3,0),(6,2,1)$, and $(6,1,0)$ for the positive-gradient curves and $(6,2,1),(6,1,0)$ and $(6,0,1)$ for the negative-gradient curves. The values $\alpha=0.2, \Delta_{z}=0.02$, and $\beta$ $=1.4$ have been considered.

out to be the lowest energy excitations. Figures 1 and 2 show the excitation energy as a function of $\Delta_{t}$ for the four different GS phases existing along the $\Delta_{t}$ energy variation line for $N$ $=6$ and 7. In all the cases the angular momentum is preserved, and a simultaneous spin and parity flip takes place. That is to say,

$$
\omega=E_{1}\left(M, S_{z} \pm 1,-P\right)-E_{1}\left(M, S_{z}, P\right),
$$

where $E_{1}\left(M, S_{z}, P\right)$ is the GS energy at $\Delta_{t}$ and $E_{1}\left(M, S_{z}\right.$ $\pm 1,-P)$ is the lowest eigenenergy within the excited configuration at the same $\Delta_{t}$. These excitations provide the lowest value $(\Delta M=l=0)$ of the multipole dispersion relation $\omega(l)$ given below.

Figures 3 and 4 show the dispersion relation of the two different types of multipole modes $\omega(l)$ mentioned previously within the third main point. In both cases, the lowest energy eigenstate within each subspace is considered. The

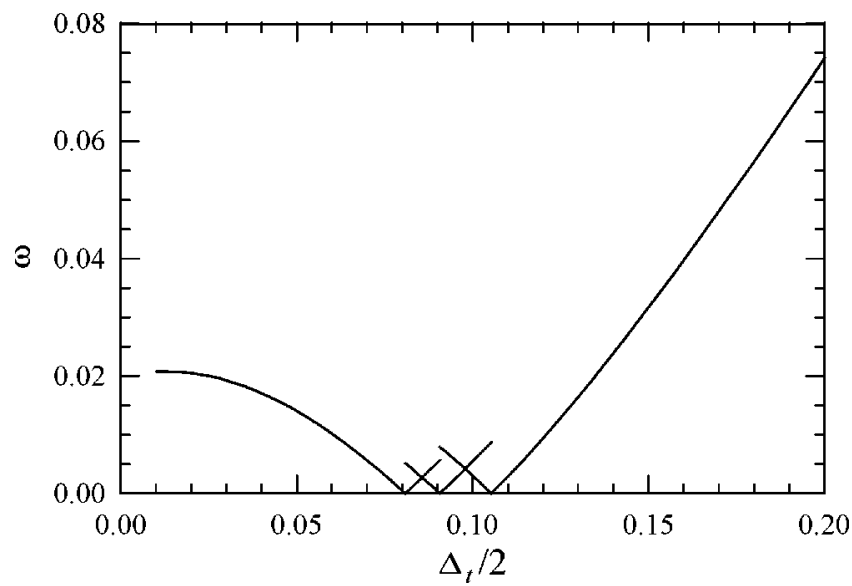

FIG. 2. The same as Fig. 1 for $N=7$. The excited configurations are (from left to right) $(9,7 / 2,0),(9,5 / 2,1)$, and $(9,3 / 2,0)$ for the positive-gradient curves and $(9,5 / 2,1),(9,3 / 2,0)$, and $(9,1 / 2,1)$ for the negative-gradient curves.

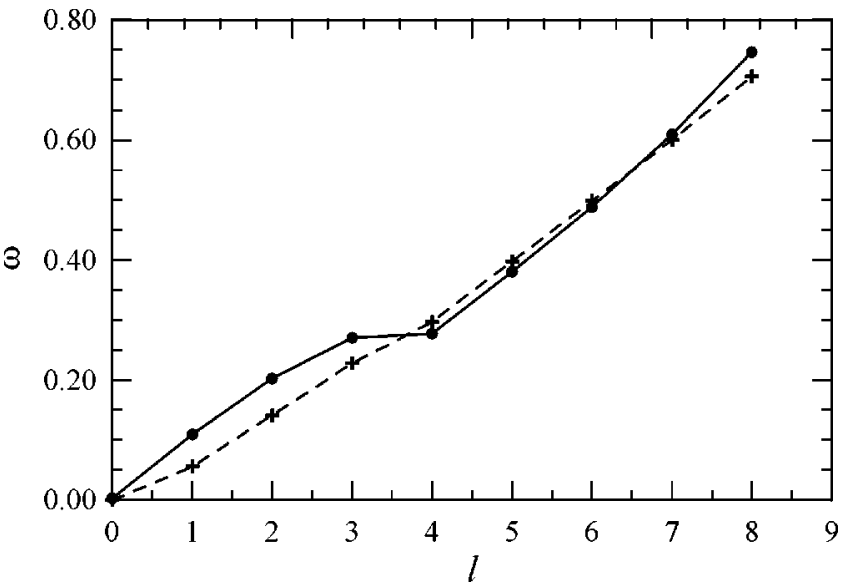

FIG. 3. Dispersion relation $\omega(l)$ of two different excited states: $(6+l, 3,0)$ (solid line) and $(6+l, 2,1)$ (dashed line) for $N=6$ and $\Delta_{t}=0.07$. The GS is the first canted state. The same values of $\alpha, \beta$, and $\Delta_{z}$ as in Fig. 1 have been used.

values of $\Delta_{t}$ were chosen in such a way that for the two systems the GS is the first canted state: $(6,2,1)$ for $N=6$ and $(9,5 / 2,1)$ for $N=7$. The general trend is given by the dashed line which corresponds to

$$
\omega=E_{1}\left(M+l, S_{z}, P\right)-E_{1}\left(M, S_{z}, P\right) .
$$

The increase of the angular momentum in one unit increases the kinetic energy of the system by $\alpha(\alpha=0.2)$. This increase is partially compensated for by a decrease of the Coulomb energy due to expansion. For both systems, the Coulomb contribution is numerically, for the particular values of the parameters that we have taken, about $2 \alpha / 3$, in such a way that the total amount of energy gained in each step is about $\alpha / 3$. This gives a quite monotonic behavior.

In contrast, unexpected features were obtained for excitations of the type (solid line),

$$
\omega=E_{1}\left(M+l, S_{z}+1,-P\right)-E_{1}\left(M, S_{z}, P\right),
$$

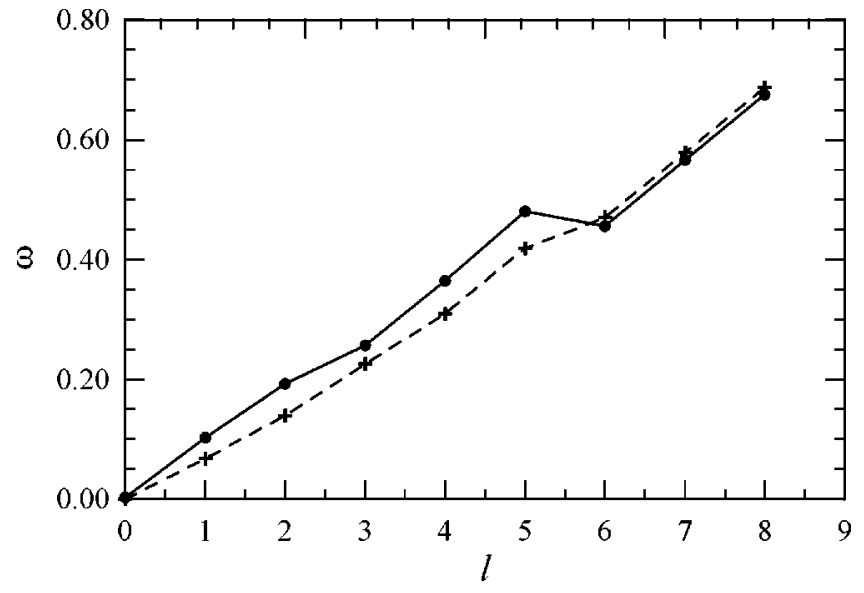

FIG. 4. The same as in Fig. 3 for the excited states: (9 $+l, 7 / 2,0)$ (solid line) and $(9+l, 5 / 2,1)$ (dashed line) for $N=7$ and $\Delta_{t}=0.085$. 
where spin and parity flips take place simultaneously. For low values of $l$, the contribution of the Coulomb energy to $\omega$ is given by a more or less constant amount of, numerically, $\alpha / 2$. However, after several steps, four for $N=6$ and six for $N=7$ the system suffers a sudden expansion that drastically reduces the Coulomb interaction. In the $N=7$ case, due to the presence of an unpaired electron, the amount of the Coulomb contribution is so large that the total energy decreases. Looking at the occupancies of the single particle states of the excited systems, the nature of the sudden change becomes clear. For ferromagnetic excited states of the type (6 $+l, 3,0)$ for $N=6$ and $(9+l, 7 / 2,0)$ for $N=7$, only spin-up states can exist. The occupied single particle states are $|s \uparrow\rangle$ and $|a \uparrow\rangle$. As $l$ increases from $l=0$, there is a slow transfer of electrons from $|a \uparrow\rangle$ to $|s \uparrow\rangle$. The sudden change takes place when the following structure is possible: $N-1$ electrons in $|s \uparrow\rangle$ with $m=0, \ldots, N-2$ and one electron in $|a \uparrow\rangle$ with $m=0$, which means $M=10(6+4)$ for $N=6$ and $M$ $=15(9+6)$ for $N=7$. For this eigenstate almost all the weight is in a single Slater determinant which means that the system is nearly uncorrelated.

The unexpected result is in clear contrast with the result obtained for a single QD at the magic values of the angular momentum, where all the energy is absorbed by the CM. In the DQD most of the absorbed energy is transformed into internal energy releasing the electrons from their interaction.

\section{DISCUSSION AND CONCLUSIONS}

We have analyzed several types of low energy excitations over the three possible GS's of a DQD confined by parabolic potentials in each plane and separated by a distance $d$. The LLL regime was considered, and the input parameters were chosen in such a way that the filling factor of the ground states and some of the excited states is close to $\nu=2$.

From the study of several dipole excitations $(\Delta M=l$ $=1$ ) we verify that the Kohn theorem is preserved in a DQD if the incident homogeneous electric field is directed along the plane of the dots. For systems with an odd number of electrons, additional excitations independent of the Coulomb interaction, such as the one induced by $E_{\perp}$, are possible. This last possibility is due to the fact that, in this case, the isospin change for a single electron does not change the Coulomb energy of the system, although it is not a consequence of the Kohn theorem.

The lowest energy excitations are characterized by a simultaneous spin and parity flip keeping the angular momentum unchanged [spin-density-waves (SDW's) for $l=0$, with energy defined by $\omega$; see Figs. 1 and 2]. They turn out to be the appropriate excitations which become degenerated with the GS at the transition boundaries in the phase diagram.

Due to the extra degree of freedom (as compared with a single dot or a single layer), represented by the isospin states and due to the interplay between tunneling and Coulomb interaction, the energy $\omega$ has a Coulomb contribution. This is in contrast to the case of a single layer for which, in the limit $k \rightarrow 0$ the noninteracting contribution given by $\Delta_{z}$ is recovered.
The softening of the $\omega$ modes with the variation of $\Delta_{t}$ shows phase instabilities previously detected in the determination of the GS phase diagram from exact diagonalization. ${ }^{1}$ There are some similarities and some differences between our results and those reported by Das Sarma et al. ${ }^{7}$ within a HF calculation for a double layer system. We will follow the arguments given by these authors to analyze our results. Within the $|\mathrm{FM}\rangle$ and $|\mathrm{SYM}\rangle$ phases, the structure of the curves $\omega / \Delta_{t}$ is similar, in both cases the $\omega$ mode softens as it approaches the phase transition from $|\mathrm{FM}\rangle$ to $|C\rangle$ and from $|\mathrm{SYM}\rangle$ to $|C\rangle$, respectively. In addition, the increase of $\omega$ as it moves away from the boundary is larger in the symmetric phase in both calculations. However, within the canted state we obtain finite values of $\omega$, albeit much smaller than those in the $|\mathrm{FM}\rangle$ or $|\mathrm{SYM}\rangle$ phases, in contrast to the results obtained in the DL for which $\omega=0$ over the full canted phase. Aside from the previous outcomings, there is another main difference: the canted state in our calculation is an eigenstate of the $S_{z}$ operator, whereas this is not the case for the HF canted phase. At the boundaries separating different phases, however, due to energy degeneracy, the superposition of states of different well defined spin gives rise to states which are not eigenstates of $S_{z}$. Das Sarma et al. ${ }^{7}$ proved that the existence of a gapless mode is directly due to the canted antiferromagnetic spin ordering. As a consequence, even though the canted states in a DQD may be interpreted as having antiferromagnetic order in the plain of the dots, ${ }^{1}$ the order is not complete, producing gapped excitations probably due to edge effects. The exceptions are at the boundaries separating different phases where due to degeneracy, the gapless mode is recovered.

Finally, unexpected nearly uncorrelated states have been found (see Figs. 3 and 4), produced by SDW excitations over the canted ground states, that develop a sudden expansion, leaving the system in a ferromagnetic state close to $\nu=1$. In addition, the structure developed by the sudden expansion on the dispersion relation at a particular value of $l$ is understood as a consequence of the existence of an intrinsic length scale in the system, related to the antiferromagnetic spin ordering in plane. The structure is well defined in the SDW dispersion relation (solid line in Figs. 3 and 4), and only slightly apparent in the charge-density-wave curve (dashed line in Figs. 3 and 4) due to the fact that within the LLL, a local variation of the spin is coupled to a variation of the density, ${ }^{5}$ producing a larger effect when spin and charge density fluctuations are induced simultaneously by the excitation. No structure was found in similar dispersion relations of SDW's over the ferromagnetic or symmetric phases. In these last cases the dispersion is given by nearly parabolic smooth curves whereas, over the canted states, in the long-wavelength limit, the dispersion relation is found to be linear, consistent with the fact that it describes antiferromagnetic fluctuations.

Within the HF calculation performed by Das Sarma for DL systems, the SDW dispersion relation does not have this type of structure. This difference may be related to the different role that the kinetic contribution to the energy plays in extended and in confined systems.

Finally, in the limit $l \rightarrow \infty$ the dispersion relation of the 
SDW does not approach asymptotically a constant value as it is the case for a single layer (SL) or DL systems due to the fact that at this limit the excitation energy always recovers the single particle value. For SL or DL systems this energy is given by $\omega^{\prime}+V_{e x}$, where $\omega^{\prime}$ is the noninteracting excitation value (a combination of $\Delta_{z}$ and $\Delta_{t}$ ) and $V_{e x}$ is the exchange single particle energy of an electron in the GS, independent of the linear momentum $k$ due to the degeneracy existing in extended systems. In contrast, in a DQD the parabolic potential breaks the degeneracy producing an increase of energy with increasing angular momentum. This gives a nearly linear curve as $l \rightarrow \infty$ typical of a single particle.

No total spin or space correlations in the ground states have been investigated through the density-correlation functions, which may signal additional symmetry breaking effects. Symmetry breaking effects of this kind have been found previously in the literature for QD's, DQD's (a vertical arrangement), and QD molecules (a horizontal arrangement) within unrestricted Hartree-Fock calculations for low magnetic fields, ${ }^{13,14}$ and within Hamiltonian diagonalization for high magnetic fields ${ }^{15}$. Whereas a comparison with the case of low magnetic fields is not possible because we have strong magnetic fields which project the system to the LLL, it would be interesting to see if our method, which includes spin degrees of freedom and does not require any truncation, shows symmetry breaking effects similar to Ref. 15 . This is left for future investigations.

\section{ACKNOWLEDGMENTS}

We gratefully acknowledge C. Tejedor and L. MartínMoreno for helpful discussions and for the code used for the Hamiltonian diagonalization. This work has been performed under Grants No. BFM2002-01868, No. FPA2001-3598 from MCyT and Feder (Spain), and Nos. 2001SGR-0064 and 2001SGR-00065 from Generalitat de Catalunya.

\section{APPENDIX}

For simplicity we neglect spin indices since they are irrelevant for the following discussion. We characterize the first quantized wave function of the $N$ particle system as $\Psi_{a_{1}, \ldots, a_{N}}\left(\mathbf{x}_{1}, \ldots, \mathbf{x}_{\mathbf{N}}\right)$, where $a_{i}=1,2$ are layer indexes indicating the dot in which the $i$ th electron sits. Hence the first quantized Hamiltonian is a matrix in layer-index space $H$

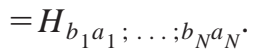

An in-plane homogeneous electric field clearly produces a contribution proportional to the identity matrix in the layer space,

$$
H_{E_{\|}} \sim \sum_{i=1}^{N} \mathbf{x}_{\mathbf{i}} \mathbf{E}_{\|}\left(\prod_{i=1}^{N} \delta_{b_{i} a_{i}}\right)
$$

and hence the interaction depends only on the center of mass coordinates, namely the Kohn theorem applies. A homogeneous electric field perpendicular to the plane $E_{\perp}$ produces a contribution diagonal in layer space (but not proportional to the identity matrix) which reads

$$
H_{E_{\perp}} \sim \sum_{i=1}^{N} \frac{d}{2}\left(\delta_{a_{i} 1}-\delta_{a_{i} 2}\right) E_{\perp}\left(\prod_{i=1}^{N} \delta_{b_{i} a_{i}}\right),
$$

where $d$ is the distance between the two dots.

The hopping term is not diagonal in layer space,

$$
H_{h o p} \sim \Delta_{t} \sum_{i=1}^{N}\left(\prod_{j=1}^{i-1} \delta_{b_{j} a_{j}}\right) s_{b_{i} a_{i}}\left(\prod_{j=i+1}^{N} \delta_{b_{j} a_{j}}\right),
$$

where $s_{11}=s_{22}=0$ and $s_{12}=s_{21}=1$. Then it is easy to check that

$$
\left[H_{E_{\perp}}, H_{h o p}\right] \sim \Delta_{t} \frac{d}{2} E_{\perp} \sum_{i=1}^{N}\left(\prod_{j=1}^{i-1} \delta_{b_{j} a_{j}}\right) \epsilon_{b_{i} a_{i}}\left(\prod_{j=i+1}^{N} \delta_{b_{j} a_{j}}\right),
$$

where $\epsilon_{b_{i} a_{i}}$ is the antisymmetric tensor. Hence a homogeneous electric field perpendicular to the plane changes the dynamics in the layer space in a nontrivial way. Since the latter is entangled with the relative motion through the Coulomb term, we conclude that a homogeneous electric field perpendicular to the plane may produce transitions between different states with the same center of mass quantum numbers; that is, the Kohn theorem does not apply.
${ }^{1}$ L. Martin-Moreno, L. Brey, and C. Tejedor, Phys. Rev. B 62, R10 633 (2000).

${ }^{2}$ B. Partoens and F. M. Peeters, Phys. Rev. Lett. 84, 4433 (2000).

${ }^{3}$ C. Tejedor, B. Paredes, J. H. Oaknin, and L. Martin-Moreno, Solid State Commun. 117, 133 (2001).

${ }^{4}$ J. H. Oaknin, L. Martin-Moreno, and C. Tejedor, Phys. Rev. B 54, 16850 (1996).

${ }^{5}$ K. Moon, H. Mori, Kun Yang, S. M. Girvin, A. H. MacDonald, L. Zheng, D. Yoshioka, and Shou-Cheng Zhang, Phys. Rev. B 51, 5138 (1995).

${ }^{6} \mathrm{~S}$. M. Girvin and A. H. MacDonald in Perspectives in the Quantum Hall Effect, edited by Das Sarma and A. Pinczuk (Wiley, New York, 1997).

${ }^{7}$ S. Das Sarma, S. Sachdev, and L. Zheng, Phys. Rev. B 58, 4672 (1998).
${ }^{8}$ S. L. Sondhi, A. Karlhede, S. A. Kivelson, and E. H. Rezayi, Phys. Rev. B 47, 16419 (1993).

${ }^{9}$ B. Paredes, C. Tejedor, L. Brey, and L. Martin-Moreno, Phys. Rev. Lett. 83, 2250 (1999).

${ }^{10}$ W. Kohn, Phys. Rev. 123, 1242 (1961).

${ }^{11}$ L. Jacak, P. Hawrylak, and A. Wjs, Quantum Dots (SpringerVerlag, Berlin, 1998).

${ }^{12}$ L. Brey, N. F. Johnson, and B. J. Halperin, Phys. Rev. B 40, 10647 (1989).

${ }^{13}$ C. Yannouleas and U. Landman, Phys. Rev. Lett. 82, 5325 (1999).

${ }^{14}$ C. Yannouleas and U. Landman, Eur. Phys. J. D 16, 373 (2001).

${ }^{15}$ M. Rontani, G. Goldoni, F. Manghi, and E. Molinari, Europhys. Lett. 58, 555 (2002). 